\title{
An arc-analytic function with nondiscrete singular set
}

\author{
by Krzysztof Kurdyka (Le Bourget-du-Lac and Kraków)
}

\begin{abstract}
We construct an arc-analytic function (i.e. analytic on every real-analytic arc) in $\mathbb{R}^{2}$ which is analytic outside a nondiscrete subset of $\mathbb{R}^{2}$.

Let $M$ be a real-analytic manifold. A function $f: M \rightarrow \mathbb{R}$ is called arc-analytic iff for every analytic arc $\gamma:]-\varepsilon, \varepsilon[\rightarrow M$ the composition $f \circ \gamma$ is analytic (see $[\mathrm{K} 1]$ ). For every function $f$ on $M$, let Sing $f$ denote the set of points of nonanalyticity of $f$. If $f$ is an arc-analytic function with subanalytic graph, then Sing $f$ is a subanalytic subset of $M$ (see [T] or [K2]); moreover, $\operatorname{dim}(\operatorname{Sing} f) \leq \operatorname{dim} M-2$ (see [K1]). Actually, in this case a stronger result is true: there exists a locally finite composition $\pi$ of local blowing-ups of $M$ such that $f \circ \pi$ is analytic (see $[\mathrm{BM}],[\mathrm{P}]$ ). Recently examples of arc-analytic functions with nonsubanalytic graphs were given ([K3] and $[\mathrm{BMP}]$, where a discontinuous example is given).

Suppose that $f: \mathbb{R}^{2} \rightarrow \mathbb{R}$ is an arc-analytic function. Professor Siciak asked whether Sing $f$ is always discrete. By the previous remarks this is the case if, for example, $f$ has subanalytic graph, because Sing $f$, being subanalytic of dimension 0 , contains only isolated points.

In this note we construct an arc-analytic function $f: \mathbb{R}^{2} \rightarrow \mathbb{R}$ such that Sing $f$ is nondiscrete and $f$ is unbounded at each point of Sing $f$. Our construction is based on an idea of [K3].
\end{abstract}

The author is grateful to Professor Siciak for stating the problem.

Let $a_{\nu} \in \mathbb{R}, \nu \in \mathbb{N}, \nu \geq 1$, be a convergent sequence in $\mathbb{R}$. Define

$$
a_{0}=\lim _{\nu \rightarrow \infty} a_{\nu}, \quad Z_{0}=\left\{\left(a_{\nu}, 0\right) \in \mathbb{R}^{2}: \nu \in \mathbb{N}, \nu \geq 0\right\} .
$$

We will construct an arc-analytic function $f: \mathbb{R}^{2} \rightarrow \mathbb{R}$ such that $\operatorname{Sing} f=$ $Z_{0}$, and $f$ is unbounded at each point of $Z_{0}$.

1991 Mathematics Subject Classification: Primary 32B20; Secondary 32B30.

Key words and phrases: arc-analytic, blow-up, projective limit, strict transform. 
We will blow up every point of $Z_{0}$ infinitely many times. To get a formal construction we take the projective limit of the following system.

Set $c_{\nu}=\left(a_{\nu}, 0\right), P_{0}=\{y=0\} \subset \mathbb{R}^{2}$

(i) Let $X_{0}=\mathbb{R}^{2}$, and let $\pi_{1,0}: X_{1} \rightarrow X_{0}$ be the blowing-up of $c_{0}$ in $X_{0}$. Let $P_{1}$ be the strict transform of $P_{0}$. We put

$$
c_{0}^{1}=\pi_{1,0}^{-1}\left(c_{0}\right) \cap P_{1} \quad \text { and } \quad c_{\nu}^{1}=\pi_{1,0}^{-1}\left(c_{\nu}\right) \quad \text { for } \nu \geq 1
$$

(we assume that $c_{\nu} \neq c_{\mu}$ for $\nu \neq \mu$ ).

(ii) Suppose we have already constructed $\pi_{n, n-1}: X_{n} \rightarrow X_{n-1}$, and $P_{n}$ is the strict transform of $P_{n-1}$ by $\pi_{n, n-1}$. Suppose we also have a sequence $c_{\nu}^{n} \in P_{n}$ such that $\pi_{n, n-1}\left(c_{\nu}^{n}\right)=c_{\nu}^{n-1}, \nu \in \mathbb{N}$. We define $\pi_{n+1, n}$ to be the compositon

$$
X_{n}^{n} \stackrel{p_{n}^{n-1}}{\longrightarrow} X_{n}^{n-1} \stackrel{p_{n}^{n-2}}{\longrightarrow} \ldots \stackrel{p_{n}^{1}}{\longrightarrow} X_{n}^{1} \stackrel{p_{n}^{0}}{\longrightarrow} X_{n}^{0}=X_{n}
$$

where each $p_{n}^{i}: X_{n}^{i+1} \rightarrow X_{n}^{i}, i=0, \ldots, n-1$, is the blowing-up of $\left(p_{n}^{i-1} \circ \ldots\right.$ $\left.\ldots \circ p_{n}^{0}\right)^{-1}\left(c_{i}^{n}\right)$ in $X_{n}$. We put $X_{n+1}=X_{n}^{n}$ and

$$
\pi_{n+1, n}=p_{n}^{n-1} \circ \ldots \circ p_{n}^{0} .
$$

Finally, let $P_{n+1}$ be the strict transform of $P_{n}$ by $\pi_{n+1, n}$ and let

$$
c_{\nu}^{n+1}=\left(\pi_{n+1, n}\right)^{-1}\left(c_{\nu}^{n}\right) \cap P_{n+1}, \quad \nu \in \mathbb{N} .
$$

We define $Z_{n}=\left\{c_{\nu}^{n} \in X_{n}: \nu \in \mathbb{N}\right\}$. For every $n \in \mathbb{N}$ we put $\pi_{n, n}=\operatorname{id}_{X_{n}}$, and for $m \leq n$ we put

$$
\pi_{n, m}=\pi_{n, n-1} \circ \ldots \circ \pi_{m+1, m} .
$$

Hence we have constructed a projective system $\pi_{n, m}: X_{n} \rightarrow X_{m}, m \leq$ $n$, with a subsystem $Z_{n} \rightarrow Z_{m}$. Then there exist topological spaces $X=$ $\underset{\lim }{\longleftarrow} X_{n}, Z=\lim Z_{n}$ and continuous mappings $\mathrm{pr}_{n}: X \rightarrow X_{n}$ such that $\mathrm{pr}_{m}=\pi_{n, m} \circ \overleftarrow{\operatorname{pr}_{n}}$ for $m \leq n$.

Set $L=X \backslash Z$. Clearly $L$ is a Hausdorff $\sigma$-compact topological space. We will define a structure of a real-analytic manifold on $L$.

Let $x=\left(x_{n}\right)_{n \in \mathbb{N}} \in L$. Then there exists an open neighbourhood $U$ of $x$ in $L$ and $n_{0} \in \mathbb{N}$ such that if $y=\left(y_{n}\right) \in U$ then $y_{k}=\operatorname{pr}_{k}(y) \notin Z_{k}$ for $k \geq n_{0}$. Hence $\left.\operatorname{pr}_{k}\right|_{U}: U \rightarrow \operatorname{pr}_{k}(U)$ is a homeomorphism. Notice that $\operatorname{pr}_{k}(U)$ is an open subset of the real-analytic manifold $X_{k}$. Clearly the family of all such mappings defines a structure of a real-analytic manifold on $L$. Moreover, each $\operatorname{pr}_{k}$ is analytic on $L$. Notice also that

$$
\operatorname{pr}_{n}: L \backslash \operatorname{pr}_{n}^{-1}\left(Z_{n}\right) \rightarrow X_{n} \backslash Z_{n}
$$

is an analytic diffeomorphism. In the sequel we need $\operatorname{pr}_{0}^{-1}: \mathbb{R}^{2} \backslash Z_{0} \rightarrow L$, which we denote by $q$. The mapping $q$ has the following property: 
LEMmA. Let $\gamma:]-\varepsilon, \varepsilon\left[\rightarrow \mathbb{R}^{2}\right.$ be an analytic arc such that $\gamma(t) \notin P_{0}=$ $\{y=0\}$ for $t \neq 0$. Then the mapping

$$
q \circ \gamma:]-\varepsilon, 0[\cup] 0, \varepsilon[\rightarrow L
$$

extends to an analytic mapping from $]-\varepsilon, \varepsilon[$ to $L$.

Proof. If $\gamma(0) \notin Z_{0}$ then the assertion is trivial. Suppose that $\gamma(0)=c_{\nu_{0}}$ for some $\nu_{0} \in \mathbb{N}$. The order of contact of $\gamma(]-\varepsilon, \varepsilon[)$ and $P_{0}=\{y=0\}$ at $\gamma(0)$ is finite. If we blow up the point $\gamma(0)$, then either the strict transforms of those curves are disjoint or the order of their contact decreases by 1 . Hence for some $n \in \mathbb{N}$,

$$
\lim _{t \rightarrow 0}\left(\pi_{n, 0}\right)^{-1} \circ \gamma(t)=\widetilde{\gamma}_{n}(0) \notin P_{n} .
$$

Clearly $\widetilde{\gamma}_{n}=\left(\pi_{n, 0}\right)^{-1} \circ \gamma$ has an analytic extension through 0 . Since

$$
q \circ \gamma=\operatorname{pr}_{n}^{-1} \circ\left(\pi_{n, 0}\right)^{-1} \circ \gamma
$$

and $\operatorname{pr}_{n}^{-1}$ is analytic outside $Z_{n}$ (recall that $Z_{n} \subset P_{n}$ ), it follows that $q \circ \gamma$ extends to a function analytic at 0 . This ends the proof of the lemma.

Recall that our analytic manifold $L$ has a countable basis of topology, hence by the Grauert Embedding Theorem ([G]) there exists a proper analytic embedding $\varphi: L \rightarrow \mathbb{R}^{N}$ for some $N \in \mathbb{N}$.

Take now a countable subset $A$ of $P_{0} \backslash Z_{0}$ which is discrete in $\mathbb{R}^{2} \backslash Z_{0}$ and $\bar{A} \backslash A=Z_{0}$. Notice that for every $n \in \mathbb{N}$ the set $\pi_{n, 0}^{-1}(A)$ is also discrete in $X_{n} \backslash Z_{n}$; moreover, $\operatorname{pr}_{n}$ maps homeomorphically $L \backslash \operatorname{pr}_{n}^{-1}\left(Z_{n}\right)$ onto $X_{n} \backslash Z_{n}$. Hence $q^{-1}(A)$ is also discrete in $L=\lim _{\longleftarrow} X_{n} \backslash \longleftarrow Z_{n}$. Thus the set $\widetilde{A}=$ $\varphi\left(q^{-1}(A)\right)$ is discrete in $\mathbb{R}^{N}$, since $\varphi$ is proper.

We claim that there exists a discrete subset $\widetilde{B}$ of $\varphi(L)$ such that if we set $B=\operatorname{pr}_{0} \circ \varphi^{-1}(\widetilde{B})$ then

$$
B \cap P_{0}=\emptyset \quad \text { and } \quad \bar{B} \backslash B=Z_{0} .
$$

To get such a $\widetilde{B}$ let us arrange the elements of $\widetilde{A}$ in a sequence $\widetilde{a}_{k}, k \in \mathbb{N}$. Notice that $\varphi\left(\operatorname{pr}_{0}^{-1}\left(P_{0}\right)\right)$ is nowhere dense in $\varphi(L)$. Hence there exists a sequence

$$
\widetilde{b}_{k} \in \varphi(L) \backslash \varphi\left(\operatorname{pr}_{0}^{-1}\left(P_{0}\right)\right)
$$

such that $\left\|\widetilde{a}_{k}-\widetilde{b}_{k}\right\|<1 / k$. We put $B=\left\{b_{0}, b_{1}, \ldots\right\}$, where $b_{k}=\operatorname{pr}_{0} \circ \varphi^{-1}\left(\widetilde{b}_{k}\right)$. Let us write, in coordinates in $\mathbb{R}^{2}, b_{k}=\left(x_{k}, y_{k}\right)$. Notice that $y_{k} \neq 0$ for all $k \in \mathbb{N}$.

Now take an analytic function $\widetilde{h}: \mathbb{R}^{N} \rightarrow \mathbb{R}$ such that $\widetilde{h}\left(\widetilde{b}_{k}\right)=y_{k}^{-2}$. Such an $\widetilde{h}$ exists since $\varphi(L)$ is closed in $\mathbb{R}^{\mathbb{N}}$, hence $\widetilde{B}$ is discrete in $\mathbb{R}^{N}$. Now put $h=\widetilde{h} \circ \varphi \circ q$ and observe that $h$ is analytic in $\mathbb{R}^{2} \backslash Z_{0}$. Finally, put

$$
f(x, y)= \begin{cases}y h(x, y) & \text { if }(x, y) \notin Z_{0} \\ 0 & \text { if }(x, y) \in Z_{0}\end{cases}
$$


To see that $f$ is arc-analytic take an analytic arc $\gamma:]-\varepsilon, \varepsilon\left[\rightarrow \mathbb{R}^{2}\right.$. If $\gamma(]-\varepsilon, \varepsilon[) \subset P_{0}=\{y=0\}$ then $f \circ \gamma \equiv 0$ is analytic. Otherwise the set $\gamma^{-1}\left(Z_{0}\right)$ is discrete in $]-\varepsilon, \varepsilon[$ and by the lemma $q \circ \gamma$ extends to an analytic mapping from $]-\varepsilon, \varepsilon[$ to $L$. Hence also $h \circ \gamma$ extends to an analytic mapping on $]-\varepsilon, \varepsilon[$. Thus $f \circ \gamma$ is analytic on $]-\varepsilon, \varepsilon[$.

Clearly $f$ is analytic in $\mathbb{R}^{2} \backslash Z_{0}$. Observe that $f\left(b_{k}\right)=f\left(x_{k}, y_{k}\right)=y_{k}^{-1}$ and $\lim _{k \rightarrow \infty} y_{k}=0$. Since $\bar{B} \backslash B=Z_{0}$, for every $\left(x_{0}, y_{0}\right) \in Z_{0}$ we have

$$
\limsup _{(x, y) \rightarrow\left(x_{0}, y_{0}\right)}|f(x, y)|=+\infty \text {. }
$$

This proves that $\operatorname{Sing} f=Z_{0}$.

Remark. This example raises two questions about arc-analytic functions.

1) Can one find an arc-analytic function on a manifold $M$ such that Sing $f$ is dense in the analytic Zariski topology (i.e. every analytic function vanishing on Sing $f$ must vanish on $M)$ ?

2) Given an arc-analytic function $f: M \rightarrow \mathbb{R}$, can one find a countable composition $\pi$ of blowing-ups such that $f \circ \pi$ is analytic? Here countable composition might be understood as a projective limit as in our example.

\section{References}

[BM] E. Bierstone and P. D. Milman, Arc-analytic functions, Invent. Math. 101 (1990), 411-424.

[BMP] E. Bierstone, P. D. Milman and A. Parusiński, A function which is arcanalytic but not continuous, Proc. Amer. Math. Soc. 113 (1991), 419-423.

[G] H. Grauert, On Levi's problem and the imbedding of real-analytic manifolds, Ann. of Math. 68 (1958), 460-472.

[K1] K. Kurdyka, Points réguliers d'un sous-analytique, Ann. Inst. Fourier (Grenoble) 38 (1) (1988), 133-156.

[K2] —, Ensembles semi-algébriques symétriques par arcs, Math. Ann. 282 (1988), $445-462$.

[K3] -, A counterexample to subanalyticity of an arc-analytic function, Ann. Polon. Math. 55 (1991), 241-243.

[P] A. Parusiński, Subanalytic functions, Trans. Amer. Math. Soc., to appear.

[T] M. Tamm, Subanalytic sets in the calculus of variations, Acta Math. 146 (1981), 167-199.

LABORATORIE DE MATHÉMATIQUES UNIVERSITÉ DE SAVOIE

CAMPUS SCIENTIFIQUE

73376 LE BOURGET-DU-LAC CEDEX, FRANCE.

E-mail: KURDYKA@UNIV-SAVOIE.FR

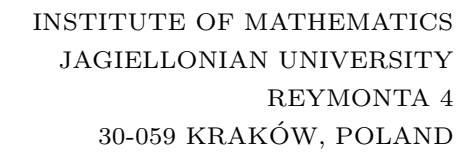

E-mail: KURDYKA@IM.UJ.EDU.PL 